\title{
POSITIVE HAUSDORFF DIMENSIONAL SPECTRUM FOR THE CRITICAL ALMOST MATHIEU OPERATOR
}

\author{
BERNARD HELFFER, QINGHUI LIU, YANHUI QU, AND QI ZHOU
}

\begin{abstract}
We show that there exists a dense set of frequencies with positive Hausdorff dimension for which the Hausdorff dimension of the spectrum of the critical almost Mathieu operator is positive.
\end{abstract}

\section{INTRODUCTION}

1.1. The context. In this paper, we are interested in the Hausdorff dimension of the spectrum of the almost Mathieu operator $H_{\lambda, \alpha, \theta}$ in $\ell^{2}(\mathbb{Z})$ (denoted from now on by AMO):

$$
\ell^{2}(\mathbb{Z}) \ni u \mapsto\left(H_{\lambda, \alpha, \theta} u\right)_{n}=u_{n+1}+u_{n-1}+2 \lambda \cos (2 \pi(n \alpha+\theta)) u_{n},
$$

where $\theta \in \mathbb{T}$ is the phase, $\alpha \in(0,1) \backslash \mathbb{Q}$ is the frequency and $\lambda \in \mathbb{R} \backslash\{0\}$ is the coupling constant. The AMO was first introduced by Peierls [24], as a model for an electron on a 2D lattice, submitted to a homogeneous magnetic field $[13,25]$. This model has been extensively studied not only because of its importance in physics $[7,23,28]$, but also as a fascinating mathematical object.

The spectrum of $H_{\lambda, \alpha, \theta}$ is a compact perfect set in $\mathbb{R}$ and, observing that it is independent of $\theta$ since $\alpha$ is irrational, we denote it by $\Sigma_{\lambda, \alpha}$. For any $\lambda$, and for any irrational $\alpha, \Sigma_{\lambda, \alpha}$ is a Cantor set [2]. Each finite interval of $\mathbb{R} \backslash \Sigma_{\lambda, \alpha}$ is called an open gap and we know from $[3,5]$ that if $\lambda \neq 1$, then for any irrational $\alpha$, all the spectral gaps are open, as predicted by the Gap Labelling Theorem [19].

However, little was known for the structure of $\Sigma_{\lambda, \alpha}$ when $\lambda=1 . H_{1, \alpha, \theta}$ is called the critical almost Mathieu operator (or Harper's equation in physics); it has particular importance in physics. One useful way to study $\Sigma_{\lambda, \alpha}$ is by periodic approximation $[6,20,21]$, through finer estimates of $\Sigma_{\lambda, p_{n} / q_{n}}$, where $p_{n}(\alpha) / q_{n}(\alpha)$ is the best rational approximation of $\alpha$, Last [21] shows that if $\alpha$ is not of bounded type, then $\Sigma_{1, \alpha}$ has zero Lebesgue measure. Finally, by the renormalization technique, Avila and Krikorian [1] completed the proof that $\Sigma_{1, \alpha}$ has zero measure, and hence a Cantor set, for all irrational 
$\alpha$. Therefore, it is natural to study the fractal dimensions of $\Sigma_{1, \alpha}$. It was believed until the mid 1990's that the box-counting $\operatorname{dimension}_{\operatorname{dim}}\left(\Sigma_{1, \alpha}\right)$ equals to $\frac{1}{2}$ for almost every $\alpha$; one can consult $[9,11,27]$ for numerical and heuristic arguments supporting this conjecture. But in 1994, WilkinsonAustin [30] provided numerical evidence that $\operatorname{dim}_{B}\left(\Sigma_{1, \alpha}\right)=0.498$ for $\alpha=\frac{\sqrt{5}-1}{2}$ and thus conjectured that $\operatorname{dim}_{B}\left(\Sigma_{1, \alpha}\right)<\frac{1}{2}$ for every irrational $\alpha$. However, Jitomirskaya-Zhang [18] showed that if $\beta(\alpha)>0$, then $\operatorname{dim}_{B}\left(\Sigma_{1, \alpha}\right)=$ 1 , which disproved Wilkinson-Austin's conjecture. Here, $\beta(\alpha)$, which measures how Liouvillean $\alpha$, is defined as

$$
\beta(\alpha):=\limsup _{n \rightarrow \infty} \frac{\log q_{n+1}(\alpha)}{q_{n}(\alpha)},
$$

where $q_{n}(\alpha)$ is the denominator of the $n$-th convergent of $\alpha$.

We recall that the Hausdorff dimension of a set $S \subset \mathbb{R}$ is defined by

$$
\operatorname{dim}_{\mathrm{H}}(S)=\inf \left\{t \in \mathbb{R}^{+} \mid \lim _{\delta \rightarrow 0} \inf _{\delta \text {-covers }} \sum_{n}\left(\operatorname{meas}\left(U_{n}\right)\right)^{t}<\infty\right\},
$$

where a $\delta$-cover of $S$ is a family $\left(U_{n}\right)_{n}$ such that $S \subset \cup_{n=1}^{\infty} U_{n}$, and every $U_{n}$ is an interval of length smaller than $\delta$. Last [21] showed that if $q_{n+1}(\alpha)>q_{n}^{4}(\alpha)$ for a subsequence of $n$, then $\operatorname{dim}_{\mathrm{H}}\left(\Sigma_{1, \alpha}\right) \leq \frac{1}{2}$. We note that the set of such kind of frequencies is a dense $G_{\delta}$ set which contains $\{\alpha \in(0,1) \backslash \mathbb{Q} \mid \beta(\alpha)>0\}$. Shamis-Last [22] showed that there exists a dense set of $\{\alpha \in(0,1) \backslash \mathbb{Q} \mid \beta(\alpha)>0\}$, for which $\operatorname{dim}_{H}\left(\Sigma_{1, \alpha}\right)=0$. Recently, Avila-Shamis-Last-Zhou [4] strengthened the result of [22] and showed that for any $\alpha$ in $\{\alpha \in(0,1) \backslash \mathbb{Q} \mid \beta(\alpha)>0\}, \operatorname{dim}_{\mathrm{H}}\left(\Sigma_{1, \alpha}\right)=0$. As we can see, the results of $[4,18,21,22]$ are more specific to Liouvillean frequency, it is interesting to see if we can say something about the Diophantine frequencies.

1.2. Main results. In this paper, we will show the following:

Theorem 1.1. The set of frequencies

$$
\mathscr{F}:=\left\{\alpha \in(0,1) \backslash \mathbb{Q}: \operatorname{dim}_{\mathrm{H}}\left(\Sigma_{1, \alpha}\right)>0\right\}
$$

is dense in $(0,1) \backslash \mathbb{Q}$ and has positive Hausdorff dimension.

Remark 1.2. Combining Theorem 1.1 with a result of [4], one thus know that $\mathscr{F}$ is dense in $\{\alpha \in(0,1) \backslash \mathbb{Q} \mid \beta(\alpha)=0\}$. 
Indeed, Bellissard ${ }^{1}$ conjectured that there should exist some $\kappa \in(0,1 / 2]$ such that $\operatorname{dim}_{\mathrm{H}}\left(\Sigma_{1, \alpha}\right)=\kappa$ for almost every $\alpha$. As far as we know, Theorem 1.1 is the first result which shows the existence of positive Hausdorff dimension of the spectrum for the critical almost Mathieu operator.

In fact, we can say more about the frequency and the lower bound of the Hausdorff dimension. Denote the continued fraction expansion of $\alpha$ as:

$$
\alpha=\frac{1}{a_{1}+\frac{1}{a_{2}+\frac{1}{a_{3}+\ldots}}}:=\left[a_{1}, a_{2}, a_{3}, \cdots\right]
$$

and define

$$
A^{*}(\alpha):=\limsup _{n \rightarrow \infty} \frac{\sum_{i=1}^{n} a_{i}}{n} \quad \text { and } \quad G_{*}(\alpha):=\liminf _{n \rightarrow \infty}\left(\prod_{i=1}^{n} a_{i}\right)^{1 / n}
$$

In 1994, Wilkinson-Austin [30] gave some numerical and heuristic arguments showing that if $n$ is large enough, then $\operatorname{dim}_{B}\left(\Sigma_{1, \alpha_{n}}\right)$ approaches $\frac{\log 2}{\log n}$, where

$$
\alpha_{n}=[n, n, n, \cdots] .
$$

Motivated by this paper, we will show the following:

Theorem 1.3. Fix $M>0$ and $\widehat{m} \in \mathbb{N}$ s.t. $\widehat{m} \geq 2$. There exist constants $C>1$ and $C^{\prime}>0$ such that for any $\alpha=\left[a_{1}, a_{2}, a_{3}, \cdots\right]$ with, for some $m \in\{0, \cdots, \widehat{m}\}$,

$$
1 \leq a_{i} \leq M,(1 \leq i \leq m) ; \quad a_{i} \geq C,(i>m)
$$

we have

$$
\operatorname{dim}_{\mathrm{H}}\left(\Sigma_{1, \alpha}\right) \geq \frac{1}{C^{\prime}} \frac{\log G_{*}(\alpha)}{A^{*}(\alpha)} .
$$

Consequently, if $n \geq C$, then, for $\alpha_{n}$ defined by (3),

$$
\operatorname{dim}_{\mathrm{H}}\left(\Sigma_{1, \alpha_{n}}\right) \geq \frac{1}{C^{\prime}} \frac{\log n}{n} .
$$

Remark 1.4. Now considering Theorem 1.3 and the results of $[4,22]$, it is reasonable to conjecture that if $A^{*}(\alpha)=\infty$, then $\operatorname{dim}_{H}\left(\Sigma_{1, \alpha}\right)=0$.

\footnotetext{
1 Private conversation with Y. Last, circ. 1995.
} 


\section{The COVERING STRUCTURE OF THE SPECTRUM: MAIN STATEMENT}

Assume $\alpha=\left[a_{1}, a_{2}, a_{3}, \cdots\right]$. Helffer and Sjöstrand [15, 16] exhibited a fine covering structure of the spectrum for a special class of frequencies. In the following, we describe a reformulation of this statement using the coding language just for the convience of the proof, readers can consult the appendix for its original formulation. Before to explain the general construction we detail the two first steps.

In the 0 -th step, there are $q_{m}(\alpha)$ disjoint bands. We code them by

$$
J_{1}, J_{2}, \cdots, J_{q_{m}(\alpha)} \text {. }
$$

Thus

$$
\Theta_{0}=\left\{1,2, \cdots, q_{m}(\alpha)\right\}
$$

is the set of words to code the bands of 0-generation.

In the first step, for each band $J_{i}$, there are finitely many sub-bands inside it. For the mid-band, we code it by $J_{i \cdot 0}$. Here $\cdot$ means the concatenation of words. On the left, there are $m_{i}$ sub-bands with good estimates on the band-length, which we code by $J_{i \cdot\left(-m_{i}\right)}, \cdots J_{i \cdot(-1)}$. Similarly, on the right, there are $n_{i}$ sub-bands, which we code by $J_{i \cdot 1}, \cdots, J_{i \cdot n_{i}}$. Write

$$
\mathcal{A}_{i}=\left\{-m_{i}, \cdots,-1,1, \cdots, n_{i}\right\}
$$

(here $\mathcal{A}$ means alphabet), then $\left\{i \cdot j: j \in \mathcal{A}_{i}\right\}$ is the set of words to code the subbands inside $J_{i}$. Now if we collect the words for all $i \in \Theta_{0}$, we obtain $\Theta_{1}$ :

$$
\Theta_{1}=\left\{i \cdot j: i \in \Theta_{0}, j \in \mathcal{A}_{i}\right\} .
$$

Since for each $i \in \Theta_{0}$, there is only one mid-band $J_{i \cdot 0}$ inside $J_{i}$,

$$
\Omega_{1}=\left\{i \cdot 0: i \in \Theta_{0}\right\}
$$

is the set of words to code the mid bands of 1-generation.

We now define inductively two sequences of words $\Theta_{k}, \Omega_{k}$ as follows. Assume $\Theta_{k}$ and $\Omega_{k}$ have been defined for some $k \geq 1$. For any $\theta \in \Theta_{k}$, fix a vector $\left(m_{\theta}, n_{\theta}\right) \in \mathbb{N}^{*} \times \mathbb{N}^{*}\left(\right.$ where $\left.\mathbb{N}^{*}=\mathbb{N} \backslash\{0\}\right)$ and write

$$
\mathcal{A}_{\theta}:=\left\{-m_{\theta}, \cdots,-1,1, \cdots, n_{\theta}\right\} .
$$

Define

$$
\Theta_{k+1}:=\left\{\theta \cdot i: \theta \in \Theta_{k} ; i \in \mathcal{A}_{\theta}\right\}
$$




$$
\Omega_{k+1}:=\Omega_{k} \cup\left\{\theta \cdot 0: \theta \in \Theta_{k}\right\}
$$

Write $\Omega_{0}=\emptyset$. Define

$$
\Theta:=\bigcup_{k \geq 0} \Theta_{k} \text { and } \Omega:=\bigcup_{k \geq 0} \Omega_{k} .
$$

As continuation of [15], the following theorem is proved in [16], the readers can just consult the appendix for its original statement.

Theorem 2.1. Fix $\widehat{m} \in \mathbb{N}$, and $M \geq 2$. Then there exist $\epsilon_{1}>0$ and, for $0<\epsilon_{0} \leq \epsilon_{1}$, some constants $C_{1}>0, b_{2}>b_{1}>0, c_{1}>0, d_{2}>d_{1}>0$ such that if $\alpha=\left[a_{1}, a_{2}, a_{3}, \cdots\right]$ and for some $0 \leq m \leq \widehat{m}$

$$
\left\{\begin{array}{c}
1 \leq a_{\ell} \leq M, \quad \ell \leq m \\
a_{\ell} \geq C_{1}, \quad \ell \geq m+1
\end{array},\right.
$$

then there exists a sequence $\left\{\left(m_{\theta}, n_{\theta}\right): \theta \in \Theta\right\}$ with

$b_{1} a_{k+m} \leq m_{\theta} \leq b_{2} a_{k+m}$ and $b_{1} a_{k+m} \leq n_{\theta} \leq b_{2} a_{k+m}, \forall k \geq 1, \forall \theta \in \Theta_{k-1}$,

and a family of bands

$$
\left\{J_{\theta}: \theta \in \Omega \cup \Theta\right\}
$$

such that:

(i) For each $k \geq 0,\left\{J_{\theta}: \theta \in \Omega_{k} \cup \Theta_{k}\right\}$ is a covering of $\Sigma_{1, \alpha}$ :

$$
\Sigma_{1, \alpha} \subset \bigcup_{\theta \in \Omega_{k} \cup \Theta_{k}} J_{\theta} .
$$

(ii) For each $k \geq 1$ and $\theta \in \Theta_{k-1}$,

$$
\partial J_{\theta} \subset \Sigma_{1, \alpha}
$$

For each $i \in \mathcal{A}_{\theta} \cup\{0\}$,

$$
J_{\theta \cdot i} \subset J_{\theta},
$$

$J_{\theta \cdot(i+1)}$ is on the right of $J_{\theta \cdot i}$. Moreover,

$$
\frac{c_{1}}{a_{k+m}} \leq \frac{d\left(J_{\theta \cdot(i+1)}, J_{\theta \cdot i}\right)}{\left|J_{\theta}\right|} .
$$

(iii) For each $k \geq 1$ and $\theta \in \Theta_{k-1}$,

$$
\frac{\left|J_{\theta \cdot 0}\right|}{\left|J_{\theta}\right|} \leq \epsilon_{0} ; \quad e^{-d_{2} a_{k+m}} \leq \frac{\left|J_{\theta \cdot i}\right|}{\left|J_{\theta}\right|} \leq e^{-d_{1} a_{k+m}},\left(i \in \mathcal{A}_{\theta}\right) .
$$




\section{HARPER'S MODEL AND SEMI-CLASSICAL ANALYSIS}

3.1. Harper's model in the rational case. We refer also to the survey of J. Bellissard [8] for a state of the art in 1991. When $\alpha$ is irrational, an equivalent way (observing that in this case $\Sigma_{\lambda, \alpha}=\cup_{\theta} \Sigma_{\lambda, \alpha, \theta}$ ) for the analysis of the spectrum in the case of a square lattice is to consider the so-called Harper model, which this time is defined on $\ell^{2}\left(\mathbb{Z}^{2}, \mathbb{C}\right)$ by

$$
\left(H^{\gamma} u\right)_{m, n}:=u_{m+1, n}+u_{m-1, n}+e^{i \gamma m} u_{m, n+1}+e^{-i \gamma m} u_{m, n-1},
$$

where $\gamma$ denotes the flux of the constant magnetic field through the fundamental cell of the lattice.

When $\alpha:=\frac{\gamma}{2 \pi}$ is a rational, Floquet theory permits to show that the spectrum is the union of the spectra of a family of $q \times q$ matrices $M_{p, q}\left(\theta_{1}, \theta_{2}\right)$ depending on the quasi-momenta $\theta=\left(\theta_{1}, \theta_{2}\right) \in \mathbb{R}^{2}$. In this way, we get that the spectrum is the union of $q$ bands $\left[\gamma_{\ell}, \delta_{\ell}\right]$ with $\delta_{\ell} \leq \gamma_{\ell+1}$, with strict inequality, except when $q$ is even for $\ell=\frac{q}{2}$. More precisely, when $\gamma=2 \pi p / q$, where $p \in \mathbb{Z}$ and $q \in \mathbb{N}^{*}$ are relatively prime, the two following matrices in $M_{q}(\mathbb{C})$ play an important role:

$$
J_{p, q}=\operatorname{diag}\left(e^{i(j-1) \gamma}\right),
$$

and

$$
\left(K_{q}\right)_{j k}=1 \text { if } k \equiv j+1[q], 0 \text { else. }
$$

In the case of Harper, the family of matrices is

$$
M_{p, q}\left(\theta_{1}, \theta_{2}\right)=e^{i \theta_{1}} J_{p, q}+e^{-i \theta_{1}} J_{p, q}^{*}+e^{i \theta_{2}} K_{q}+e^{-i \theta_{2}} K_{q}^{*} .
$$

The Chambers formula gives a very elegant formula for this determinant:

$$
\operatorname{det}\left(M_{p, q}\left(\theta_{1}, \theta_{2}\right)-\lambda\right)=f_{p, q}(\lambda)+(-1)^{q+1} 2\left(\cos q \theta_{1}+\cos q \theta_{2}\right),
$$

where $f_{p, q}$ is a polynomial of degree $q$. Each band $I_{\ell}$ is described by a solution $\lambda_{\ell}\left(\theta_{1}, \theta_{2}\right)$ of the Chambers equation which can be expressed in the form

$$
\lambda_{\ell}\left(\theta_{1}, \theta_{2}\right)=\varphi_{\ell, p, q}\left(2\left(\cos q \theta_{1}+\cos q \theta_{2}\right)\right) .
$$

3.2. Semi-classical analysis. It can be shown that the spectrum $\Sigma_{\lambda, \alpha}$ is the same as the spectrum of the operator $\widehat{H}_{\gamma}$ acting on $L^{2}(\mathbb{R})$ defined by

$$
L^{2}(\mathbb{R}) \ni u \mapsto \lambda\left(\tau_{\gamma}+\tau_{-\gamma}\right) u+2 \cos x u \in L^{2}(\mathbb{R}),
$$

where $\tau_{\gamma}$ is defined by $\tau_{\gamma} u(x)=u(x-\gamma)$.

In other words, $\widehat{H}_{\gamma}$ is the Weyl's $\gamma$-quantization of the symbol $(x, \xi) \mapsto$ $2(\lambda \cos \xi+\cos x)$. Let us recall what is meant by this. The symbols are $C^{\infty}$ 
functions of $\mathbb{R}^{2} \ni(x, \xi) \mapsto p(x, \xi, h)$ which are $C^{\infty}$ bounded (in our case they are in addition $(2 \pi)$-periodic in each variable) depending on a semi-classical parameter $\gamma=h \in\left[-h_{0}, 0\right) \cup\left(0, h_{0}\right], h_{0}>0$ (view as "little") and satisfying

$$
\forall(j, k) \in \mathbb{N}^{2} ; \exists C_{j, k} ; \forall(x, \xi) \in \mathbb{R}^{2},\left|\partial_{x}^{j} \partial_{\xi}^{k} p(x, \xi, h)\right| \leq C_{j, k} .
$$

The Weyl quantization of the symbol $p$ (for $h \neq 0,|h| \leq h_{0}$ ) is the pseudodifferential operator acting on $L^{2}(\mathbb{R})$ by

$$
\mathrm{Op}_{h}^{W}(p) u(x)=\frac{1}{2 \pi h} \iint e^{i(x-y) \xi / h} p\left(\frac{x+y}{2}, \xi, h\right) u(y) d y d \xi .
$$

An important fact is that when $p$ is real valued the associate operator is self-adjoint. This approach is only powerful when $h$ is small. This is what is call semi-classical analysis. Hence, this leads as to analyze the spectrum near $\alpha=0$.

3.3. Semi-classical analysis near $\alpha=0$ when $\lambda=1$. Here we have to analyze the spectrum of the $h$-pseudodifferential operator of symbol $p(x, \xi):=$ $2(\cos x+\cos \xi)$, i.e. $2\left(\cos x+\cos h D_{x}\right)=2 \cos x+\left(\tau_{h}+\tau_{-h}\right)$. For $E \in$ $[-4,4] \backslash\{0\}$, semi-classical analysis says that one has first to look at the energy level $p(x, \xi)=E$. Here we see that the energy level is the union of curves which can be indexed by $\mathbb{Z}^{2}$. Modulo $\mathcal{O}\left(h^{\infty}\right)$, the spectrum is obtained by looking at a Hamiltonian has a symbol $\tilde{p}(x, \xi)$ such that $\tilde{p}(x, \xi) \rightarrow+\infty$ and $\tilde{p}^{-1}(E-\epsilon, E+\epsilon)=p^{-1}(E-\epsilon, E+\epsilon) \cap(-\pi, \pi)^{2}$. For this operator the spectrum is discrete and is given near $E$ by the Bohr-Sommerfeld quantization which determines a sequence of eigenvalues $\lambda_{k}(h)$ in $\left(-4,-\epsilon_{0}\right) \cup\left(\epsilon_{0}, 4\right)$, whose asymptotic is known modulo $\mathcal{O}\left(h^{\infty}\right)$. Coming back to the initial problem, the analysis of the tunneling between the different wells leads to a localization of the spectrum in a family of intervals whose center is $\mathcal{O}\left(h^{\infty}\right)$ close to the sequence $\lambda_{k}(h)$ and whose size is exponentially small and can be measured precisely.

The next step is that in each of these intervals the spectral analysis of the restriction of our initial operator appears to be the $h^{\prime}$-quantization of an operator whose symbol is close (after renormalization) to $\cos \xi+\cos x$ and where $\frac{h^{\prime}}{2 \pi} \equiv \frac{2 \pi}{h}$ (modulo $\mathbb{Z}$ ). This relation gives a strong link between the corresponding continued fraction expansions of $\alpha=\frac{h}{2 \pi}$ and $\alpha^{\prime}=\frac{h^{\prime}}{2 \pi}$ since $\frac{h^{\prime}}{2 \pi}=\left[a_{2}, a_{3}, \cdots\right]$. Under our assumption $h^{\prime}$ is small (because $a_{2}$ is assumed to be large) and we can redo the same analysis leading again to a new familly of intervals. Of course, this is quite technical to control the uniformity of the constants appearing in the renormalization procedure. In the first step 


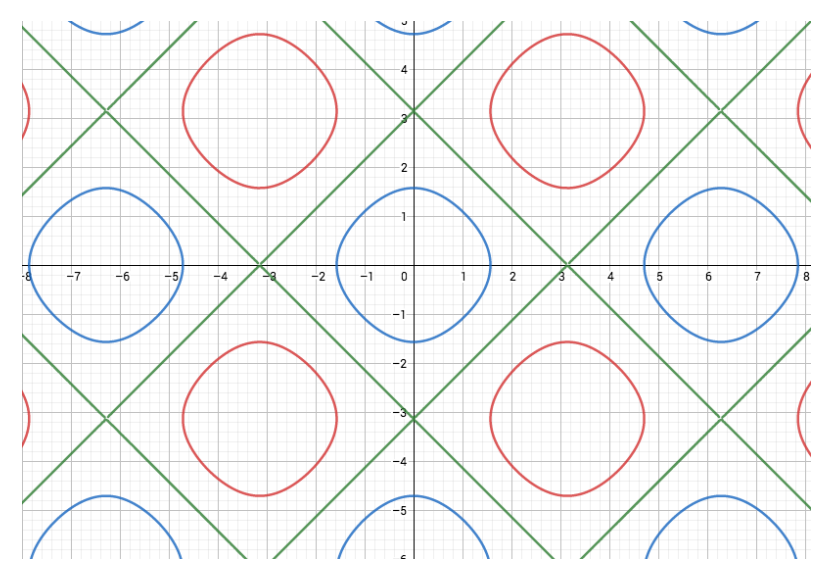

FiguRE 1. Energy levels for $(x, \xi) \mapsto \cos x+\cos \xi$. In Green $E=0$, in blue $E=1$, in $\operatorname{red} E=-1$.

$E=0$ corresponds to critical values of $p(x, \xi)$ with a saddle point. The analysis near this point is much more involved and the introduction of this $\epsilon_{0}$ permits to avoid this analysis in our paper like in [16].

3.4. Semi-classical analysis near a rational. When $\alpha=\frac{p}{q}+\hbar$, one can show that $\Sigma_{\alpha, 1}$ is the spectrum of the Weyl $h$-quantization of $M_{p, q}(x, \xi)$ (with $h=2 \pi \hbar$ ). Hence we have again to perform a semi-classical analysis but this time for a system of $h$-pseudodifferential operator. As shown by Chamber's formula (10), the semi-classical analysis for $\hbar$ small is strongly related with the semi-classical analysis of a function of $\cos q x+\cos q h D_{x}$ (see (11)). Hence, near each of the $q$ bands, we have to perform a spectral analysis which is close to the analysis of the previous subsection. When $q$ is even, there is a need for a special analysis for the two central touching bands (see Appendix).

\section{The lower bound of the Hausdorff dimension of the SPECTRUM}

In this section, we prove Theorem 1.1 and Theorem 1.3. At first we construct a Cantor subset of the spectrum based on Theorem 2.1. Then we estimate the lower bound of the Hausdroff dimension of this Cantor set. Finally, we prove the main theorems.

\subsection{A subset of the spectrum.}


Fix a frequency $\alpha=\left[a_{1}, a_{2}, \cdots\right]$ satisfying the condition in Theorem 2.1. For each $n \geq 1$, we introduce

$$
\kappa_{n}:=\left[b_{1} a_{m+n}\right],
$$

where $[x]$ denotes the integer part of the number $x, b_{1}$ is defined in Theorem 2.1 .

In the following, we will construct a Cantor subset of $\Sigma_{1, \alpha} \cap J_{1}$, where $J_{1}$ the most left interval of 0 -generation. For all $n \geq 1$, define

$$
\widehat{\Theta}_{n}:=\{1\} \times \prod_{i=1}^{n}\left\{1,2, \cdots, \kappa_{i}\right\} \quad \text { and } \quad \mathscr{C}_{n}:=\bigcup_{\theta \in \widehat{\Theta}_{n}} J_{\theta} .
$$

By (7), we have $\widehat{\Theta}_{n} \subset \Theta_{n}$. Define the set of codings as

$$
\widehat{\Theta}_{\infty}:=\{1\} \times \prod_{i=1}^{\infty}\left\{1,2, \cdots, \kappa_{i}\right\}
$$

For any $\omega=\omega_{0} \omega_{1} \omega_{2} \cdots \in \widehat{\Theta}_{\infty}$, we denote the prefix of length $n+1$ of $\omega$ by

$$
\left.\omega\right|_{n}:=\omega_{0} \cdots \omega_{n} .
$$

By Theorem $2.1, \mathscr{C}_{n}$ is a decreasing sequence of compact sets. Define

$$
\mathscr{C}:=\bigcap_{n \geq 0} \mathscr{C}_{n}
$$

By Theorem 2.1 (iii) and (6), for any $\theta \in \widehat{\Theta}_{n}$, we have

$$
\left|J_{\theta}\right|=\left|J_{1}\right| \prod_{k=1}^{n} \frac{\left|J_{\left.\theta\right|_{k}}\right|}{\left|J_{\left.\theta\right|_{k-1}}\right|} \leq\left|J_{1}\right| \prod_{k=1}^{n} e^{-d_{1} a_{k+m}} \leq\left|J_{1}\right| e^{-d_{1} C_{1} n} .
$$

Thus, $\left|J_{\theta}\right|$ tends to 0 when the length of the word $\theta$ tends to infinity. So, $\mathscr{C}$ is a Cantor set. Indeed, the definition of $\widehat{\Theta}_{n}$ make sure that the chosen band always avoid the excluded intervals of size $\approx \epsilon_{0}$ at each step.

Proposition 4.1. $\mathscr{C} \subset \Sigma_{1, \alpha} \cap J_{1}$.

Proof. By Theorem 2.1 (ii), for each $\theta \in \widehat{\Theta}_{n}$ we have $\partial J_{\theta} \subset \Sigma_{1, \alpha} \cap J_{1}$. By the construction of $\mathscr{C}$ and Theorem 2.1, for any $x \in \mathscr{C}$, there exists a unique coding sequence $\omega \in \widehat{\Theta}_{\infty}$ such that

$$
\{x\}=\bigcap_{n \geq 0} J_{\left.\omega\right|_{n}} .
$$

Let $x_{n}$ be the left endpoint of $J_{\left.\omega\right|_{n}}$, then $x_{n} \in \Sigma_{1, \alpha} \cap J_{1}$ and $x_{n} \rightarrow x$. Since $\Sigma_{1, \alpha} \cap J_{1}$ is compact, we conclude that $x \in \Sigma_{1, \alpha} \cap J_{1}$. 


\subsection{The lower bound of $\operatorname{dim}_{H} \mathscr{C}$.}

We need to use a basic fact from fractal geometry which we recall now. Assume $X \subset \mathbb{R}$ is a Borel set. Let $\mu$ be a probability measure supported on $X$. For any $x \in X$, the lower local dimension of $\mu$ at $x$ is defined by

$$
\underline{d}_{\mu}(x):=\liminf _{r \rightarrow 0} \frac{\log \mu(B(x, r))}{\log r} .
$$

Proposition 4.2 (Prop. 10.1 of [10]). If $\underline{d}_{\mu}(x) \geq d_{0}$ for $\mu$-a.e. $x \in X$, then $\operatorname{dim}_{H} X \geq d_{0}$.

For given $c_{1}>0$ and $d_{1}>0$, we introduce $\tilde{C}\left(c_{1}, d_{1}\right)>1 / d_{1}$ such that

$$
\frac{c_{1} \exp \left(d_{1} \tilde{C}\right)}{\tilde{C}}>2 \text {. }
$$

Proposition 4.3. Let $\widehat{m}, M, \epsilon_{0} \in\left(0, \epsilon_{1}\right), b_{1}, c_{1}, d_{1}, d_{2}$ be the constants given as in Theorem 2.1. Fix $m$ with $0 \leq m \leq \widehat{m}$. Assume that $\alpha=$ $\left[a_{1}, a_{2}, \cdots\right]$ satisfies $1 \leq a_{i} \leq M$ for $1 \leq i \leq m$ and $a_{i} \geq \max \left\{C_{1}, \tilde{C}\left(c_{1}, d_{1}\right)\right\}$ for $i \geq m+1$. Then

$$
\operatorname{dim}_{H} \mathscr{C} \geq \frac{\log \left(b_{1} / 2\right)+\log G_{*}(\alpha)}{d_{2} A^{*}(\alpha)} .
$$

Proof. For any $n \geq 1$, if we write

$$
\mathcal{F}_{n}:=\left\{\mathscr{C} \cap J_{\theta}: \theta \in \hat{\Theta}_{n}\right\},
$$

then $\mathcal{F}_{n}$ is a finite $\sigma$-algebra on $\mathscr{C}$. We define a measure $\mu_{n}$ on $\left(\mathscr{C}, \mathcal{F}_{n}\right)$ as

$$
\mu_{n}\left(\mathscr{C} \cap J_{\theta}\right):=\frac{1}{\prod_{i=1}^{n} \kappa_{i}} .
$$

By Carathéodory extension theorem (see for example [26]), there exists a unique probability measure $\mu$ on $\mathscr{C}$ such that $\left.\mu\right|_{\mathcal{F}_{n}}=\mu_{n}$.

Now we fix $x \in \mathscr{C}$, and will estimate $\underline{d}_{\mu}(x)$. Let $\omega \in \widehat{\Theta}_{\infty}$ be the coding of $x$, that is,

$$
\{x\}=\bigcap_{n \geq 1} J_{\left.\omega\right|_{n}} .
$$

Take $r>0$. There exists a unique $n_{r} \in \mathbb{N}$ such that

$$
J_{\left.\omega\right|_{n_{r}}} \subset B(x, r), \quad J_{\omega_{n_{r}-1}} \not \subset B(x, r) .
$$

This implies that

$$
\left|J_{\left.\omega\right|_{n_{r}}}\right|<2 r, \quad\left|J_{\left.\omega\right|_{n_{r}-1}}\right| \geq r
$$

Claim: $B(x, r)$ can only intersect one band of $\left(n_{r}-1\right)$-generation, which is 


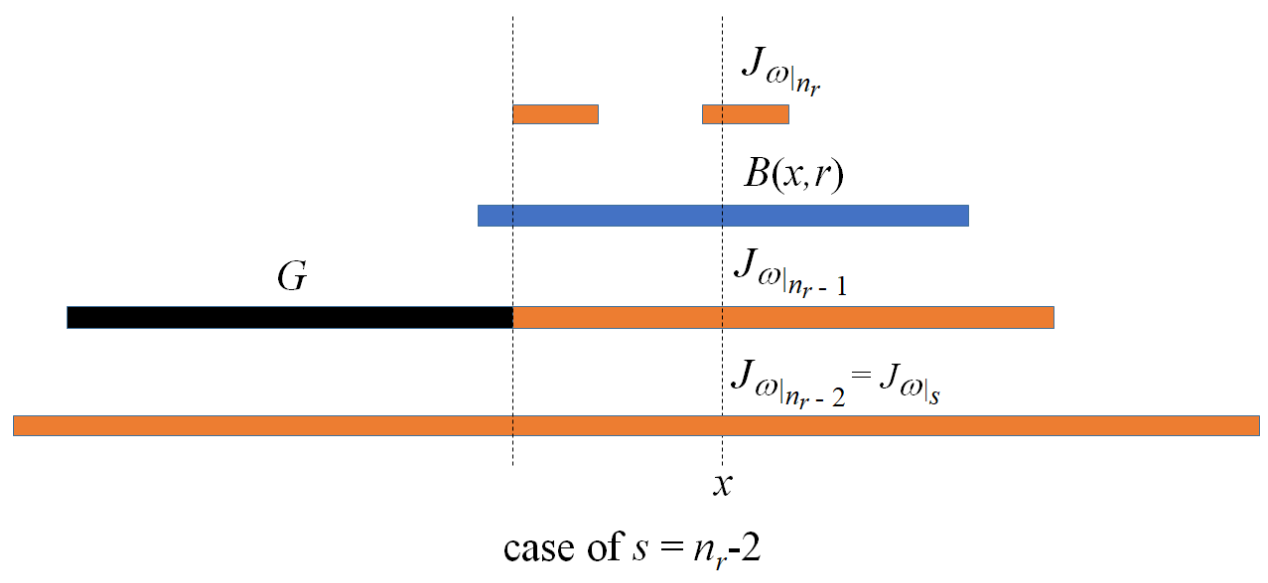

Figure 2.

$J_{\left.\omega\right|_{n_{r}-1}}$.

$\triangleleft$ If $B(x, r) \subset J_{\left.\omega\right|_{n-1}}$, then the claim holds trivially. In the following, we assume $B(x, r) \not \subset J_{\left.\omega\right|_{n_{r}-1}}$. To prove the claim, we only need to show that the lengths of the gaps in the left and right of $J_{\left.\omega\right|_{n_{r-1}}}$ are all bigger than $2 r$.

We call any component of $\operatorname{Co}(\mathscr{C}) \backslash \mathscr{C}$ a gap of $\mathscr{C}$, where $\operatorname{Co}(\mathscr{C})$ is the convex hull of $\mathscr{C}$. A gap $G$ is called of order $k$, if $G$ is a subset of some band of $k$-generation but is not a subset of any band of $(k+1)$-generation.

Denote the gap in the left of $J_{\left.\omega\right|_{n_{r}-1}}$ by $G$. By our construction of $\mathscr{C}$, $\mathrm{G}$ is a gap of order $s$ for some $s \leq n_{r}-2$ (see Figure 2 and Figure 3). That is, $G \subset J_{\theta}$ for some $\theta \in \hat{\Theta}_{s}$, but $G$ is not a subset of any band of $(s+1)$-generation.

We claim that $\theta=\left.\omega\right|_{s}$. If otherwise, there exists some $\tilde{s} \leq s$ such that $\hat{\theta}:=\theta_{0} \cdots \theta_{\tilde{s}-1}=\left.\omega\right|_{\tilde{s}-1}$ but $\theta_{\tilde{s}} \neq \omega_{\tilde{s}}$. Then $J_{\theta}$ and $J_{\left.\omega\right|_{s}}$ are two different descendants of same generation of $J_{\hat{\theta}}$, hence disjoint. In particular, $\bar{G} \cap$ $J_{\left.\omega\right|_{s}}=\emptyset$, since $\bar{G} \subset J_{\theta}$, where $\bar{G}$ is the closure of $G$. On the other hand, the right endpoint of $G$ is the left endpoint of $J_{\left.\omega\right|_{n_{r}-1}}$, thus $\bar{G} \cap J_{\left.\omega\right|_{n_{r}-1}} \neq \emptyset$. Notice that, $J_{\left.\omega\right|_{n_{r}-1}} \subseteq J_{\left.\omega\right|_{s}}$ since $s \leq n_{r}-1$. So, $\bar{G} \cap J_{\left.\omega\right|_{s}} \neq \emptyset$, which is a contradiction.

Thus $G \subset J_{\left.\omega\right|_{s}}$ is a gap of order $s$. Notice that, $J_{\left.\omega\right|_{n_{r}-1}} \subset J_{\left.\omega\right|_{s+1}}$ since $s+1 \leq n_{r}-1$. Also, since $a_{m+s+1} \geq \tilde{C}$, by Theorem 2.1 (ii), (iii), (14) and 
(15), we have

$$
|G| \geq \frac{c_{1}\left|J_{\left.\omega\right|_{s}}\right|}{a_{m+s+1}} \geq \frac{c_{1} e^{d_{1} a_{m+s+1}}\left|J_{\left.\omega\right|_{s+1}}\right|}{a_{m+s+1}} \geq 2\left|J_{\left.\omega\right|_{s+1}}\right| \geq 2\left|J_{\left.\omega\right|_{n_{r}-1}}\right| \geq 2 r .
$$

By the same argument, we can show that the gap in the right of $J_{\left.\omega\right|_{n_{r}-1}}$ also has length bigger than $2 r$. Then the claim follows.

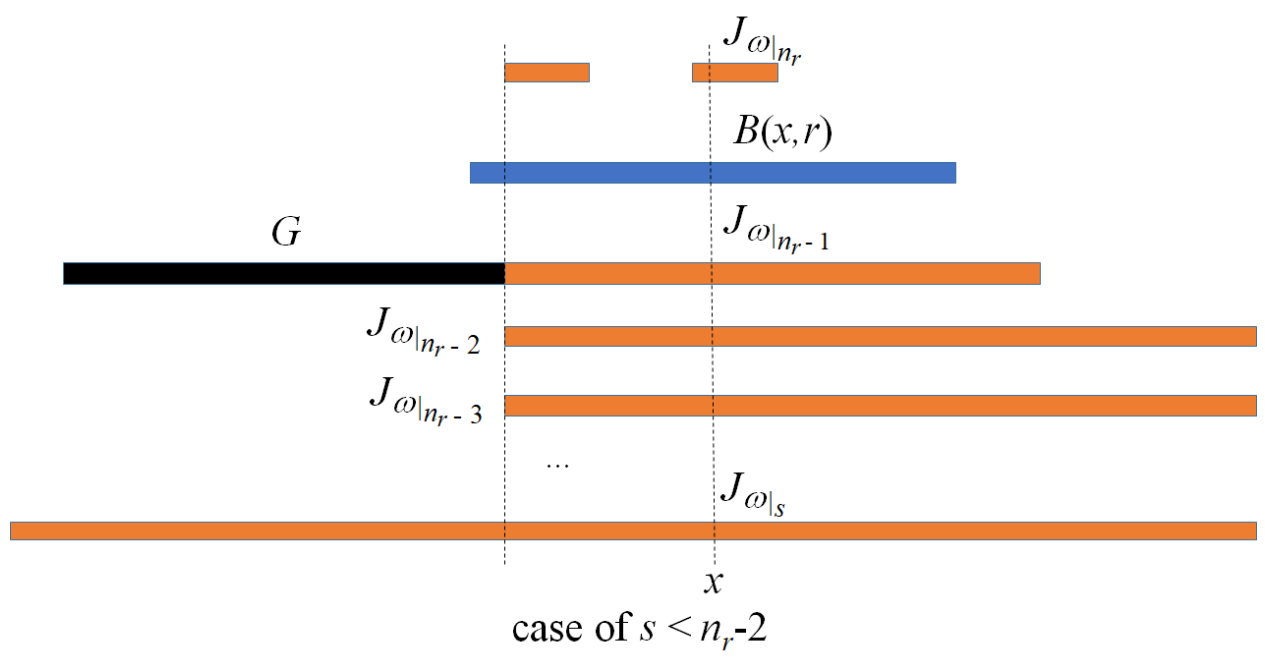

FIGURE 3.

By the claim, we have

$$
\mu(B(x, r)) \leq \mu\left(J_{\left.\omega\right|_{n_{r}-1}}\right)=\frac{1}{\prod_{i=1}^{n_{r}-1} \kappa_{i}} .
$$

By Theorem 2.1 (iii) and the assumption, we have

$$
\frac{\left|J_{\left.\omega\right|_{k}}\right|}{\left|J_{\left.\omega\right|_{k-1}}\right|} \geq e^{-d_{2} a_{m+k}}
$$

Consequently,

$$
\left|J_{\left.\omega\right|_{n}}\right|=\left|J_{1}\right| \prod_{k=1}^{n_{r}} \frac{\left|J_{\left.\omega\right|_{k}}\right|}{\left|J_{\left.\omega\right|_{k-1}}\right|} \geq\left|J_{1}\right| e^{-d_{2} \sum_{k=1}^{n_{r}} a_{m+k}} .
$$

By (15), we get

$$
\log r \geq \log \left|J_{1}\right|-\log 2-d_{2} \sum_{k=1}^{n_{r}} a_{m+k}
$$


Recall that $\kappa_{i}=\left[b_{1} a_{m+i}\right]$ and by the assumption, $a_{m+i} \geq 2 / b_{1}$. So we have $\kappa_{i} \geq b_{1} a_{m+i} / 2$. Combining (17) and (18), we get

$$
\begin{aligned}
\frac{\log \mu(B(x, r))}{\log r} & \geq \frac{\sum_{i=1}^{n_{r}-1} \log \kappa_{i}}{d_{2} \sum_{k=1}^{n_{r}} a_{m+k}+\log 2-\log \left|J_{1}\right|} \\
& \geq \frac{\left(n_{r}-1\right) \log \frac{b_{1}}{2}+\sum_{i=1}^{n_{r}-1} \log a_{m+i}}{d_{2} \sum_{k=1}^{n_{r}} a_{m+k}+\log 2-\log \left|J_{1}\right|} .
\end{aligned}
$$

By taking the lower limit, we get

$$
\underline{d}_{\mu}(x)=\liminf _{r \rightarrow 0} \frac{\log \mu(B(x, r))}{\log r} \geq \frac{\log \left(b_{1} / 2\right)+\log G_{*}(\alpha)}{d_{2} A^{*}(\alpha)} .
$$

then by Proposition 4.2, we conclude that

$$
\operatorname{dim}_{H} \mathscr{C} \geq \frac{\log \left(b_{1} / 2\right)+\log G_{*}(\alpha)}{d_{2} A^{*}(\alpha)} .
$$

This achieves the proof of Proposition 4.3.

\subsection{Proof of Theorem 1.3. Define}

$$
C:=\max \left\{C_{1}, \tilde{C}\left(c_{1}, d_{1}\right), 2 / b_{1},\left(2 / b_{1}\right)^{2}\right\} \quad \text { and } \quad C^{\prime}:=2 d_{2} .
$$

If $b_{1} \geq 2$, then by Proposition 4.1 and 4.3 ,

$$
\operatorname{dim}_{H} \Sigma_{1, \alpha} \geq \operatorname{dim}_{H} \mathscr{C} \geq \frac{\log G_{*}(\alpha)}{d_{2} A^{*}(\alpha)} \geq \frac{1}{C^{\prime}} \frac{\log G_{*}(\alpha)}{A^{*}(\alpha)} .
$$

If $b_{1}<2$, then we have $G_{*}(\alpha) \geq C \geq\left(2 / b_{1}\right)^{2}$ and consequently

$$
\log G_{*}(\alpha) \geq-2 \log \left(b_{1} / 2\right) .
$$

Then by Proposition 4.1 and 4.3, we have

$\operatorname{dim}_{H} \Sigma_{1, \alpha} \geq \operatorname{dim}_{H} \mathscr{C} \geq \frac{\log G_{*}(\alpha)+\log \left(b_{1} / 2\right)}{d_{2} A^{*}(\alpha)} \geq \frac{\log G_{*}(\alpha)}{2 d_{2} A^{*}(\alpha)}=\frac{1}{C^{\prime}} \frac{\log G_{*}(\alpha)}{A^{*}(\alpha)}$.

Thus (4) follows, and (5) is a direct consequence of (4).

4.4. Proof of Theorem 1.1. At first, we show that $\mathscr{F}$ is dense in $(0,1) \backslash \mathbb{Q}$. Let $m=\widehat{m}=M$. Let $C$ be the constant in Theorem 1.3. Define

$$
\mathscr{F}_{M}:=\left\{\alpha: a_{i} \leq M,(1 \leq i \leq M) ; a_{i}=C(i \geq M+1)\right\} .
$$

Then by Theorem 1.3, $\mathscr{F}_{M} \subset \mathscr{F}$. On the other hand, for any $\alpha, \alpha^{\prime} \in(0,1) \backslash \mathbb{Q}$, let $n$ be the first index for which the continued fraction expansions of $\alpha$ and $\alpha^{\prime}$ differ and define the distance of $\alpha$ and $\alpha^{\prime}$ as

$$
d_{H}\left(\alpha, \alpha^{\prime}\right)=\frac{1}{n+1} \text {. }
$$


Endow $(0,1) \backslash \mathbb{Q}$ with this topology, then it is easy to see that this topology coincide with the usual topology induced from $\mathbb{R}$ and $\bigcup_{M \geq 1} \mathscr{F}_{M}$ is dense in $(0,1) \backslash \mathbb{Q}$. Hence, $\mathscr{F}$ is also dense in $(0,1) \backslash \mathbb{Q}$.

Now we show that $\mathscr{F}$ has positive Hausdorff dimension. Indeed, by Theorem 1.3 , we know that

$$
\widehat{\mathscr{F}}:=\left\{\alpha \in(0,1) \backslash \mathbb{Q}: C \leq a_{n} \leq 10 C, n \geq 1\right\} \subset \mathscr{F} .
$$

By Theorem 11 of [12], $\widehat{\mathscr{F}}$ has positive Hausdorff dimension.

\section{Appendix A. The statement of Theorem 0.1 in [16]}

We give a translation from the french, correcting also a few typos and adding a few explanatory remarks.

Theorem A.1. Let $\hat{m} \in \mathbb{N}(\hat{m} \geq 2)$ and $M \geq 2$. There exists $\epsilon_{1}>0$ and, for $\epsilon_{0} \in\left(0, \epsilon_{1}\right)$, a constant $C=C\left(\hat{m}, M, \epsilon_{0}\right)>0$ such that if $\alpha=\left[a_{1}, a_{2}, \ldots,\right]$ is irrational and satisfies for some $m \leq \hat{m}$

$$
\begin{array}{ll}
1 \leq\left|a_{j}\right| \leq M & \text { for } 0<j \leq m \\
\left|a_{j}\right| \geq C & \text { for } j \geq m+1,
\end{array}
$$

then $\Sigma_{1, \alpha}$ is contained in the union of $q_{m}$ intervals $I_{\ell}(h)\left(\ell=1, \cdots, q_{m}\right)$ in the form $\left[\gamma_{\ell}(h), \delta_{\ell}(h)\right]$ with

$$
\begin{aligned}
& \gamma_{\ell}(h), \delta_{\ell}(h) \in \Sigma_{1, \alpha}, \\
& \gamma_{\ell}<\delta_{\ell} \leq \gamma_{\ell+1}<\delta_{\ell+1}, \\
& \gamma_{\ell}(h) \geq \gamma_{\ell}-C|h| \text { and } \delta_{\ell}(h)<\delta_{\ell}+C|h|, \\
& \gamma_{\ell}(h) \geq \gamma_{\ell}+\frac{1}{C} \sqrt{h} \text { if } \delta_{\ell-1}=\gamma_{\ell},
\end{aligned}
$$

where

$$
\begin{gathered}
\alpha^{(m)}=\left[a_{1}, \ldots, a_{m}\right]=\frac{p_{m}}{q_{m}}, \\
h=2 \pi\left(\alpha-\alpha^{(m)}\right), \\
\cup_{\ell}\left[\gamma_{\ell}, \delta_{\ell}\right]=\Sigma_{1, \alpha}^{(m)}, \\
d\left(I_{\ell}(h), I_{\ell+1}(h)\right) \geq \frac{1}{C} \text { if } \delta_{\ell} \neq \gamma_{\ell+1} \text { and } \geq \frac{1}{C} \sqrt{|h|} \text { if } \delta_{\ell}=\gamma_{\ell+1} .
\end{gathered}
$$

For each interval $I_{\ell}(h), \Sigma_{1, \alpha} \cap I_{\ell}(h)$ can be described as living in a union of $N_{\ell, j}$ closed intervals $J_{j}^{(\ell)}$ (indexed by $j \in\left(-m_{\ell, j}, n_{\ell, j}\right)$ ) of length $\neq 0$ with $\partial J_{j}^{(\ell)} \subset \Sigma_{1, \alpha}, J_{j+1}^{(\ell)}$ on the right of $J_{j}^{(\ell)}$ and

$$
\begin{aligned}
& m_{\ell, j} \approx\left|a_{m+1}\right| \text { and } n_{\ell, j} \approx\left|a_{m+1}\right| \\
& \frac{1}{\left|a_{m+1}\right|} \lesssim d\left(J_{j}^{(\ell)}, J_{j+1}^{(\ell)}\right) \lesssim \frac{1}{\sqrt{\left|a_{m+1}\right|}}
\end{aligned}
$$




$$
J_{0}^{(\ell)} \text { has length } 2 \epsilon_{0}+\mathcal{O}\left(\frac{1}{\left|a_{m+1}\right|}\right) .
$$

The other bands have size

$$
\exp \left(-C(j)\left|a_{m+1}\right|\right) \text { with } C(j) \approx 1 .
$$

For $j \neq 0$, if $\kappa_{j}^{(\ell)}$ is the affine function sending $J_{j}^{(\ell)}$ in $[-2,+2]$, then

$$
\kappa_{j}^{(\ell)}\left(J_{j}^{(\ell)}\right) \cap \Sigma_{1, \alpha} \subset \cup_{k} J_{j, k}^{(\ell)},
$$

where the $J_{j, k}^{(\ell)}$ have analogous properties to the $J_{j}^{(\ell)}$ with $a_{m+1}$ replaced by $a_{m+2}$ and (26) can be improved in the form

$$
d\left(J_{j, k}^{(\ell)}, J_{j, k+1}^{(\ell)}\right) \approx \frac{1}{\left|a_{m+2}\right|} .
$$

One can then iterate indefinitely.

Here in the statements $a \lesssim b$ means that $a / b \leq C$ where $C$ depends only on $C_{0}$ and $\epsilon_{0}$. The same is true when we use the notation $\mathcal{O}$ or $\approx$.

Remark A.2. $\epsilon_{0}$ corresponds with the exclusion in each interval and at each step of the renormalization of a small interval of size $\approx 2 \epsilon_{0}$ for which another analysis has to be done and which was the object of [17] (see also [14]). This corresponds to the energy 0 for the map $(x, \xi) \mapsto 2(\cos x+\cos \xi)$. This refined analysis is not needed here.

Remark A.3. The possibility of having $\delta_{\ell}=\gamma_{\ell+1}$ is due to the occurence of touching bands. Van Mouche [29] has proven that it occurs only when $q_{m}$ is even and for $\ell=\frac{q_{m}}{2}$. These two touching bands lead to the lower bound (24) and the weaker estimate in (26).

\section{Appendix B. Acknowledgements}

Q.-H. Liu was supported by NSFC grant (11571030). Y.-H. Qu was supported by NSFC grant (11431007 and 11790273). Q. Zhou was partially supported by NSFC grant (11671192), "Deng Feng Scholar Program B" of Nanjing University, Specially-appointed professor programme of Jiangsu province.

\section{REFERENCES}

[1] A. Avila and R. Krikorian, Reducibility or non-uniform hyperbolicity for quasiperiodic Schrödinger cocycles. Ann. Math. 164, 911-940 (2006).

[2] A. Avila and S. Jitomirskaya, The Ten Martini Problem, Ann. Math. 170, 303-342 (2009). 
[3] A. Avila and S. Jitomirskaya, Almost localization and almost reducibility, J. Eur. Math. Soc, 12, 93-131 (2010).

[4] A. Avila, Y. Last, M. Shamis, and Q. Zhou, On the abominable properties of the Almost Mathieu operator with well approximated frequencies, in preparation.

[5] A. Avila, J. You and Z. Zhou, The Dry Ten Martini Problem in the non-critical case, preprint.

[6] J.Avron, P. van Mouche, B. Simon, On the measure of the spectrum for the almost Mathieu operator. Commun. Math. Phys. 132, 103-118 (1990)

[7] J. E. Avron, D. Osadchy and R. Seiler, A topological look at the quantum Hall effect. Physics today. 38-42. (2003).

[8] J. Bellissard, Le papillon de Hofstadter. Astérisque 206 (1992) 7-39.

[9] J. Bell and R. B. Stinchcombe, Hierarchical band clustering and fractal spectra in incommensurate systems. J. Phys. A 20, L739-L744 (1987).

[10] K. Falconer, Techniques in fractal geometry. John Wiley \& Sons, Ltd., Chichester, 1997.

[11] T. Geisel, R. Ketzmerick and G.Petshel, New class of level statistics in quantum systems with unbounded diffusion. Phys. Rev. Lett. 66, 1651-1654 (1991).

[12] I.J. Good, The fractional dimensional theory of continued fractions. Proc. Cambridge Philos. Soc. 37, (1941). 199-228.

[13] P.G. Harper, Single band motion of conduction electrons in a uniform magnetic field, Proc. Phys. Soc. London A. 68, 874-892 (1955).

[14] B. Helffer and P. Kerdelhué. On the Total Bandwidth for the Rational Harper's Equation. Commun. Math. Phys. 173, 335-356 (1995).

[15] B. Helffer and J. Sjöstrand, Analyse semi-classique pour l'équation de Harper (avec application à l'équation de Schrödinger avec champ magnétique). Mém. Soc. Math. France, No. 34, 1-113 (1988).

[16] B. Helffer and J. Sjöstrand, Analyse semi-classique pour l'équation de Harper II: Comportement semi-classique près d'un rationnel. Mém. Soc. Math. France, No. 40, 1-139. (1990).

[17] B. Helffer and J. Sjöstrand, Semi-classical analysis for the Harper's equation III: Cantor structure of the spectrum. Mém. Soc. Math. France, No. 39. 1-124 (1989).

[18] S. Jitomirskaya and S. Zhang, Quantitative continuity of singular continuous spectral measures and arithmetic criteria for quasiperiodic Schrödinger operators. ArXiv preprint in arXiv:1510.07086 (2015).

[19] R. Johnson and J. Moser, The rotation number for almost periodic potentials. Comm. Math. Phys, 84, 403-438 (1982).

[20] I. Krasovsky. Central spectral gaps of the Almost Mathieu Operator. Commun. Math. Phys. 351, 419-439 (2017).

[21] Y. Last, Zero measure spectrum for the almost Mathieu Operator. Commun. Math. Phys. 164, 421-432 (1994).

[22] Y. Last and M. Shamis, Zero Hausdorff Dimension Spectrum for the Almost Mathieu Operator. Commun. Math. Phys. 348, 729-750 (2016).

[23] D. Osadchy and J. E. Avron, Hofstadter butterfly as quantum phase diagram. J. Math Phys. 42, 5665-5671 (2001). 
[24] R. Peierls, Zur Theorie des Diamagnetismus von Leitungselektronen. Z. Phys. 80, 763-791 (1933).

[25] A. Rauh, Degeneracy of Landau levels in crystals, Phys. Status Solidi B 65, 131-135 (1974).

[26] H. L. Royden, Real analysis. Third edition. Macmillan Publishing Company, New York, 1988.

[27] C. Tang and M. Kohmoto, Global scaling properties of the spectrum for a quasiperiodic Schrödinger equation. Phys. Rev. B 34, 2041-2044 (1986).

[28] D.J. Thouless, M. Kohmoto, M.P. Nightingale, and M. Den Nijs, Quantized Hall conductance in a two dimensional periodic potential. Phys. Rev. Lett. 49, 405-408 (1982).

[29] P. Van Mouche. The coexistence problem for the discrete Mathieu operator. Comm. Math. Phys. 122 (1989), no. 1, 23-33.

[30] M. Wilkinson and E.J. Austin, Spectral dimension and dynamics for Harper's equation. Phys. Rev. B 50, 1420-1430 (1994).

Laboratoire de Mathématiques Jean Leray, Université de NANTEs ANd CNRS, 2 Rue de la Houssinière 44322 Nantes Cedex (France) and Laboratoire de Mathématiques d'Orsay, Univ. Paris-Sud, Université Paris-Saclay.

E-mail address: Bernard.Helffer@univ-nantes.fr

Department of Computer Science, Beijing Institute of Technology, Beijing 100081, PR CHINA.

E-mail address: qhliu@bit.edu.cn

Department of Mathematics, Tsinghua University, Beijing 100084, PR China.

E-mail address: yhqu@tsinghua.edu.cn

Department of Mathematics, Nanjing University, Nanjing 210093, China

E-mail address: qizhou@nju.edu.cn 